\title{
Prevalence of Rapid Eye Movement-related Obstructive Sleep Apnea in Adult Narcolepsy
}

\author{
Tetsuro Hoshino ${ }^{1}$, Ryujiro Sasanabe ${ }^{1}$, Mamiko Mano ${ }^{1}$, Atsuhiko Nomura ${ }^{1}$, Chihiro Kato ${ }^{1}$, \\ Masako Sato ${ }^{1}$, Masato Imai ${ }^{1}$, Kenta Murotani ${ }^{2}$, Christian Guilleminault ${ }^{3}$ and Toshiaki Shiomi ${ }^{1}$
}

\begin{abstract}
:
Objective The association between narcolepsy and rapid eye movement (REM)-related obstructive sleep apnea (OSA) has not been reported. This study aimed to examine the prevalence of REM-related OSA in narcolepsy patients.

Methods From January 2013 to April 2018, 141 adult patients were diagnosed with narcolepsy using nocturnal polysomnography and the multiple sleep latency test. The prevalence of REM-related OSA in narcolepsy patients was retrospectively reviewed. Three criteria were used to determine REM-related OSA: Definition \#1, an overall apnea-hypopnea index (AHI) $\geq 5$ and AHI during REM (AHIREM)/AHI during non-rapid eye movement (NREM) (AHINREM) $\geq 2$; Definition \#2, an overall AHI $\geq 5$ and $\mathrm{AHI}_{\text {REM }} / \mathrm{AHI}_{\mathrm{NREM}} \geq 2$ and $\mathrm{AHI}_{\mathrm{NREM}}<15$; and Definition \#3, an overall $\mathrm{AHI} \geq 5$ and $\mathrm{AHI}_{\mathrm{REM}} / \mathrm{AHI}_{\mathrm{NREM}} \geq 2$ and $\mathrm{AHI}_{\mathrm{NREM}}<8$ plus an REM sleep duration $>10.5$ minutes.

Results Of the 141 narcolepsy patients, 26 were diagnosed with narcolepsy with cataplexy (NA-CA) and 115 with narcolepsy without cataplexy (NA w/o CA). Seventeen patients with NA-CA and 39 with NA w/o CA had OSA. According to Definition \#1, the prevalence of REM-related OSA was $47.1 \%$ and $41.0 \%$, respectively, in OSA patients with NA-CA and NA w/o CA; according to Definition \#2, the respective prevalence was $47.1 \%$ and $38.5 \%$, while that according to Definition \#3 was $41.2 \%$ and $25.6 \%$. No significant differences were found in the prevalence of REM-related OSA for each definition.

Conclusion A high prevalence of REM-related OSA was confirmed in adult narcolepsy patients with OSA. Compared to previous reports, we noted a high frequency of REM-related OSA satisfying the relatively strict Definition \#3. These results might reflect the pathophysiological characteristics of narcolepsy.
\end{abstract}

Key words: narcolepsy, obstructive sleep apnea, REM-related obstructive sleep apnea

(Intern Med 58: 2151-2157, 2019)

(DOI: 10.2169/internalmedicine.2601-18)

\section{Introduction}

Narcolepsy is a neurological disorder with several primary symptoms: excessive daytime sleepiness (EDS), cataplexy, and rapid eye movement (REM)-related symptoms, such as hypnagogic hallucination and sleep paralysis caused by an increased propensity for REM sleep $(1,2)$. Obesity is not a primary symptom of patients with narcolepsy; however, previous studies have noted an association between narcolepsy and an increased body mass index (BMI) (3). Furthermore, obesity is a potent risk factor of obstructive sleep apnea (OSA) (4). Therefore, narcolepsy patients tend to suffer from OSA and sometime need therapeutic intervention, such as continuous positive airway pressure (CPAP) therapy (5).

OSA is characterized by repetitive apnea and hypopnea due to total or partial collapse of the upper airway during sleep. In recent reports, OSA has often been divided into two patterns of pathophysiology: non-stage specific OSA and REM-related OSA (6). Clinically, it is important to separate OSA into these patterns because previous studies have indicated that REM-related OSA is independently asso-

${ }^{1}$ Department of Sleep Medicine and Sleep Disorders Center, Aichi Medical University Hospital, Japan, ${ }^{2}$ Center for Clinical Research, Aichi Medical University Hospital, Japan and ${ }^{3}$ Sleep Medicine Division, Stanford University School of Medicine, United States Received: December 29, 2018; Accepted: February 6, 2019; Advance Publication by J-STAGE: April 17, 2019 Correspondence to Dr. Ryujro Sasanabe, sasanabe@aichi-med-u.ac.jp 
ciated with prevalent and incident hypertension, increased insulin resistance, impairment of human spatial navigational memory, and non-dipping nocturnal blood pressure $(7,8)$. Furthermore, recent studies have reported that patients with REM-related OSA occasionally do not tolerate CPAP therapy $(6,9)$.

Several studies have described the prevalence of OSA and pathophysiological mechanisms contributing to an increased rate of OSA in narcolepsy patients $(5,10-14)$. However, the association between narcolepsy and REM-related OSA has not been reported. The aim of this study was to clarify the prevalence of REM-related OSA in adult narcolepsy patients diagnosed in our sleep disorders center.

\section{Materials and Methods}

This retrospective study was approved by the ethics committee of Aichi Medical University Hospital (AMUH). Informed consent was waived due to the non-invasive and retrospective nature of this study. We assessed 896 patients who first visited our sleep center at AMUH and underwent polysomnography (PSG) and the 5-nap multiple sleep latency test (MSLT) due to suspicion of narcolepsy, hypersomnia, or other sleep disorders from January 2013 to April 2018. A total of 190 of the 896 patients met the International Classification of Sleep Disorders (ICSD)-2 criteria for narcolepsy (15). Of these 190 patients, 49 who were <18 years of age were excluded, as the criteria of OSA are different for pediatric patients according to ICSD-2. Ultimately, 141 adult patients were enrolled in this study. At the time of the first visit, all patients filled out questionnaire, which was used to determine the Epworth sleepiness scale (ESS) and the presence of cataplexy, hypnogogic hallucination, and sleep paralysis. All patients underwent diagnostic overnight PSG followed by the 5-nap MSLT the following morning (16).

Overnight PSG was performed on an Alice 6 System (Philips Respironics, Tokyo, Japan). The following biological variables were continuously monitored: electroencephalogram (EEG), submental and anterior tibial electromyogram (EMG), electrocardiogram (ECG), bilateral electrooculogram (EOG), airflow measured by nasal pressure transducer and thermistor, respiratory effort measured by thoracic and abdominal inductive plethysmography bands, body position, snoring sound, and arterial oxygen saturation. Apnea, hypopnea, and other PSG parameters were scored manually by polysomnographic technologist according to the American Academy of Sleep Medicine (AASM) 2007, version 2.1 (17). The apnea-hypopnea index (AHI) was defined as the average number of apnea and hypopnea events per hour of sleep, and OSA was defined by an AHI $\geq 5$ according to ICSD-2 (15). According to previous reports, three criteria were used to determine REM-related OSA: Definition \#1: an overall $\mathrm{AHI} \geq 5$ and $\mathrm{AHI}$ during REM $\left(\mathrm{AHI}_{\mathrm{REM}}\right) / \mathrm{AHI}$ during non-rapid eye movement (NREM) $\left(\mathrm{AHI}_{\mathrm{NREM}}\right) \geq 2$, Definition $\# 2$ : an overall $\mathrm{AHI} \geq 5$ and $\mathrm{AHI}_{\text {REM }} / \mathrm{AHI}_{\text {NREM }} \geq 2$ and $\mathrm{AHI}_{\text {NREM }}$
$<15$ and Definition \#3: an overall $\mathrm{AHI} \geq 5$ and $\mathrm{AHI}_{\mathrm{REM}} /$ $\mathrm{AHI}_{\mathrm{NREM}} \geq 2$ and $\mathrm{AHI}_{\mathrm{NREM}}<8$ and REM sleep duration $>10.5$ minutes $(6,18,19)$.

The MSLT consisted of 5 naps performed on the day after PSG according to the standard protocol (20). Narcolepsy was diagnosed and subdivided into narcolepsy with cataplexy (NA-CA) and narcolepsy without cataplexy (NA w/o CA) according to ICSD-2 (15). In this study, none of patients underwent the measurement of their cerebrospinal fluid orexin A level, as this examination for narcoleptics is not covered by the Japanese health insurance system.

All clinical records, PSG and MSLT date were made available in anonymize format. Using these data, we evaluated the prevalence of REM-related OSA among NA-CA and NA w/o CA patients with OSA.

\section{Statistical analyses}

All continuous variables were expressed as the mean \pm standard deviation. Categorical variables were expressed as the number (percentages) and were compared using Fisher's exact test. Continuous variables were compared between the groups using Student's $t$-test for normally distributed data or the Mann-Whitney $U$ test for non-normally distributed data. Normality was assessed using the Shapiro-Wilk test. A p value $<0.05$ was considered to be statistically significant. Statistical analyses were performed using the SPSS software program for Windows, version 25.0 (SPSS, Chicago, USA).

\section{Results}

Of 141 narcolepsy patients, 26 were diagnosed with NACA and 115 with NA w/o CA. Seventeen patients with NACA and 39 with NA w/o CA had OSA (Fig. 1).

Table 1 shows demographic, polysomnographic, and MSLT findings of narcolepsy patients with and without OSA. We noted significant differences between narcolepsy patients with and without OSA in the age $(35.4 \pm 15.0$ vs. $27.6 \pm 9.8 \mathrm{p}=0.001)$, BMI $(25.6 \pm 5.2$ vs. $21.6 \pm 2.5 \mathrm{p}<0.001)$, percentage of cataplexy (30.4\% vs. $10.6 \%$, respectively; $\mathrm{p}=$ $0.003)$, sleep efficiency $(87.7 \pm 11.6$ vs. $92.1 \pm 5.9 \mathrm{p}=0.02)$, arousal index $(28.0 \pm 15.7$ vs. $16.3 \pm 7.2 \mathrm{p}<0.001), \% \mathrm{~N} 1(26.2$ \pm 15.2 vs. $21.5 \pm 18.3 \mathrm{p}=0.006), \% \mathrm{~N} 2(47.0 \pm 13.2$ vs. $51.6 \pm$ $11.3 \mathrm{p}=0.016)$, AHI $(15.0 \pm 10.1$ vs. $2.0 \pm 1.3 \mathrm{p}<0.001)$, AHIREM (22.7 \pm 15.9 vs. $3.1 \pm 2.9 \mathrm{p}=0.002)$, and AHINREM $(12.7 \pm 10.8$ vs. $1.7 \pm 1.3 \mathrm{p}=0.003)$.

Table 2 shows the characteristics, electrophysiological findings, and prevalence of REM-related OSA in the OSA patients with NA-CA and NA w/o CA. According to Definition \#1, the prevalence of REM-related OSA was $47.1 \%$ and $41.0 \%$, respectively, in OSA patients with NA-CA and NA w/o CA; according to Definition \#2, the respective prevalence was $47.1 \%$ and $38.5 \%$, while that according to Definition \#3 was $41.2 \%$ and $25.6 \%$. No significant differences were found in the prevalence of REM-related OSA for each definition. However, we did note a significant difference between OSA patients with NA-CA and NA w/o CA in the 




Figure 1. Flow diagram of the study. NA-CA: narcolepsy with cataplexy, NA w/o CA: narcolepsy without cataplexy

Table 1. Characteristics and Electrophysiological Findings in Narcolepsy Patients with and without OSA.

\begin{tabular}{lcccc}
\hline & \multicolumn{3}{c}{ Narcolepsy } \\
\cline { 2 - 5 } & total(n=141) & with OSA(n=56) & without OSA(n=85) & p value \\
\hline Age & $30.7 \pm 12.7$ & $35.4 \pm 15.0$ & $27.6 \pm 9.8$ & $0.001^{*}$ \\
Sex male (\%) & $96(68.1)$ & $42(75.0)$ & $54(63.5)$ & 0.106 \\
BMI (kg/m $)$ & $23.3 \pm 4.3$ & $25.6 \pm 5.2$ & $21.6 \pm 2.5$ & $<0.001^{*}$ \\
ESS & $14.8 \pm 5.1$ & $14.7 \pm 5.6$ & $14.8 \pm 4.8$ & 0.882 \\
Medications & & & & \\
Antihypertensive (\%) & $8(5.7)$ & $7(12.5)$ & $1(1.2)$ & $0.006^{*}$ \\
Antidepressant (\%) & $9(6.4)$ & $4(7.1)$ & $5(5.9)$ & 0.738 \\
Benzodiazepine-hypnotic (\%) & $14(9.9)$ & $7(12.5)$ & $7(8.2)$ & 0.402 \\
Cataplexy (\%) & $26(18.4)$ & $17(30.4)$ & $9(10.6)$ & $0.003^{*}$ \\
Hypnagogic hallucination (\%) & $29(20.6)$ & $14(25.0)$ & $15(17.6)$ & 0.199 \\
Sleep paralysis (\%) & $43(30.5)$ & $20(35.7)$ & $23(27.1)$ & 0.182 \\
Mean sleep latency in MSLT (min) & $2.3 \pm 1.6$ & $2.0 \pm 1.4$ & $2.5 \pm 1.7$ & 0.118 \\
Mean REM latency in MSLT (min) & $4.9 \pm 2.7$ & $4.6 \pm 2.7$ & $5.0 \pm 2.8$ & 0.411 \\
Number of SOREMP & $3.2 \pm 1.1$ & $3.1 \pm 1.2$ & $3.2 \pm 1.1$ & 0.524 \\
Sleep efficiency (\%) & $90.3 \pm 8.9$ & $87.7 \pm 11.6$ & $92.1 \pm 5.9$ & $0.02^{*}$ \\
Arousal index (events/h) & $18.5 \pm 10.4$ & $28.0 \pm 15.7$ & $16.3 \pm 7.2$ & $<0.001^{*}$ \\
\%REM (\%) & $22.6 \pm 6.0$ & $22.0 \pm 5.9$ & $22.9 \pm 6.1$ & 0.487 \\
\%N1 (\%) & $23.4 \pm 17.2$ & $26.2 \pm 15.2$ & $21.5 \pm 18.3$ & $0.006^{*}$ \\
\%N2 (\%) & $49.7 \pm 12.2$ & $47.0 \pm 13.2$ & $51.6 \pm 11.3$ & $0.016^{*}$ \\
\%N3 (\%) & $6.0 \pm 5.9$ & $5.5 \pm 5.5$ & $6.4 \pm 6.1$ & 0.288 \\
AHI (events/h) & $7.1 \pm 9.0$ & $15.0 \pm 10.1$ & $2.0 \pm 1.3$ & $<0.001^{*}$ \\
AHIREM (events/h) & $10.9 \pm 14.0$ & $22.7 \pm 15.9$ & $3.1 \pm 2.9$ & $<0.001^{*}$ \\
AHINREM (events/h) & $6.1 \pm 8.7$ & $12.7 \pm 10.8$ & $1.7 \pm 1.3$ & $0.003^{*}$ \\
AHIREM/AHINREM & $3.1 \pm 3.9$ & $3.3 \pm 3.9$ & $2.9 \pm 3.9$ & 0.398 \\
\hline
\end{tabular}

*p $<0.05$ when comparing the narcolepsy patients with and without OSA.

REM: rapid eye movement, BMI: body mass index, OSA: obstructive sleep apnea, SOREMP: sleep-onset REM sleep period, \%REM: percentage of stage REM per total sleep time, \%N1: percentage of stage 1 per total sleep time, \%N2: percentage of stage 2 per total sleep time, AHI: \%N3: percentage of stage 3 per total sleep time, AHI: apnea and hypopnea index, AHIREM: apnea and hypopnea index during the REM sleep period, AHINREM: apnea and hypopnea index during the NREM sleep period, ESS: Epworth sleepiness scale

age $(45.0 \pm 20.1$ vs. $31.2 \pm 9.9 \mathrm{p}=0.019)$, ESS (16.9 \pm 5.2 vs. $13.7 \pm 5.5 \mathrm{p}=0.025)$, mean REM sleep latency in MSLT $(2.6 \pm$ 1.7 vs. $5.5 \pm 2.7 \mathrm{p}<0.001$ ), number of SOREMP (4.0 \pm 1.2 vs. $2.7 \pm 1.0 \mathrm{p}=0.001)$, sleep efficiency $(80.1 \pm 16.1$ vs. $91.1 \pm 6.4 \mathrm{p}$ $=0.015)$, arousal index $(31.8 \pm 16.3$ vs. $20.4 \pm 9.1 \mathrm{p}=0.012), \%$ $\mathrm{N} 1(36.1 \pm 17.4$ vs. $21.9 \pm 11.9 \mathrm{p}=0.003)$ and $\% \mathrm{~N} 2(37.5 \pm 14.7$ vs. $51.3 \pm 10.0 \mathrm{p}=0.002)$.

\section{Discussion}

This is the first study evaluating the prevalence of OSA and REM-related OSA in adult narcolepsy patients. 
Table 2. Characteristics, Electrophysiological Findings, and Prevalence of REM-related OSA in Patients with NA-CA and NA w/o CA with OSA.

\begin{tabular}{lccc}
\hline & $\begin{array}{c}\text { NA-CA } \\
\text { with OSA }(\mathrm{n}=17)\end{array}$ & $\begin{array}{c}\text { NA w/o CA } \\
\text { with OSA }(\mathrm{n}=39)\end{array}$ & p value \\
\hline Age & $45.0 \pm 20.1$ & $31.2 \pm 9.9$ & $0.019^{*}$ \\
Sex male (\%) & $10(58.8)$ & $32(82.1)$ & 0.065 \\
BMI $\left(\mathrm{kg} / \mathrm{m}^{2}\right)$ & $27.1 \pm 6.3$ & $25.0 \pm 4.5$ & 0.314 \\
ESS & $16.9 \pm 5.2$ & $13.7 \pm 5.5$ & $0.025^{*}$ \\
Mean sleep latency in MSLT (min) & $1.6 \pm 1.2$ & $2.1 \pm 1.5$ & 0.205 \\
Mean REM latency in MSLT (min) & $2.6 \pm 1.7$ & $5.5 \pm 2.7$ & $<0.001^{*}$ \\
number of SOREMP (MSLT) & $4.0 \pm 1.2$ & $2.7 \pm 1.0$ & $0.001^{*}$ \\
Sleep efficiency (\%) & $80.1 \pm 16.4$ & $91.1 \pm 6.4$ & $0.015^{*}$ \\
Arousal Index (events/h) & $31.8 \pm 16.3$ & $20.4 \pm 9.1$ & $0.012^{*}$ \\
\%REM (\%) & $22.0 \pm 5.2$ & $22.1 \pm 6.2$ & 0.803 \\
\%N1 (\%) & $36.1 \pm 17.4$ & $21.9 \pm 11.9$ & $0.003^{*}$ \\
\%N2 (\%) & $37.5 \pm 14.7$ & $51.3 \pm 10.0$ & $0.002^{*}$ \\
\%N3 (\%) & $4.3 \pm 5.4$ & $6.1 \pm 5.5$ & 0.105 \\
AHI (events/h) & $16.5 \pm 10.2$ & $14.3 \pm 10.1$ & 0.387 \\
AHIREM (events/h) & $24.6 \pm 15.6$ & $21.8 \pm 16.2$ & 0.423 \\
AHINREM (events/h) & $14.0 \pm 11.7$ & $12.2 \pm 10.5$ & 0.782 \\
AHIREM/AHINREM & $3.4 \pm 3.8$ & $3.2 \pm 3.9$ & 0.682 \\
REM-related OSA definition \#1 & $8(47.1)$ & $16(41.0)$ & 0.675 \\
REM-related OSA definition \#2 & $8(47.1)$ & $15(38.5)$ & 0.548 \\
REM-related OSA definition \#3 & $7(41.2)$ & $10(25.6)$ & 0.245 \\
\hline
\end{tabular}

$* \mathrm{p}<0.05$ when comparing the group of NA-CA and NA w/o CA with OSA.

REM: rapid eye movement, NA-CA: narcolepsy with cataplexy, NA w/o CA: narcolepsy without cataplexy, BMI: body mass index, OSA: obstructive sleep apnea, SOREMP: sleep-onset REM sleep period, AHI: apnea and hypopnea index, AHIREM: apnea and hypopnea index during REM sleep period, AHINREM: apnea and hypopnea index during NREM sleep period, ESS: Epworth sleepiness scale, Definition \#1: an overall AHI $\geq 5$ and AHIREM/AHINREM $\geq 2$, Definition \#2: an overall AHI $\geq 5$ and AHIREM/AHINREM $\geq 2$ and AHINREM $<15$, Definition \#3: an overall AHI $\geq 5$ and AHIREM/AHINREM $\geq 2$ and AHINREM $<8$ and REM sleep duration $>10.5 \mathrm{~min}$

Previous studies have shown that the prevalence of OSA in narcolepsy patients ranges from $2 \%$ to $68 \%(5,10-14)$. This marked variability is due to the use of inconsistent definitions for OSA and some studies being conducted in very small groups. The definitions of OSA differ among studies, ranging from $\mathrm{AHI} \geq 5$ (10), AHI $\geq 10$ (5) to $\mathrm{AHI}$ $\geq 15$ (13). In the present study, we defined OSA as AHI $\geq 5$ according to the ICSD-2, and the prevalence of OSA among patients with narcolepsy was 39.7\%. Surprisingly, 17 of 26 NA-CA patients $(65.4 \%)$ had OSA (Fig. 1). These results suggest that not only do aging and a tendency to be obese play a role in OSA, but other pathophysiological mechanisms might also contribute to the increased prevalence of OSA in narcolepsy patients. Indeed, previous studies have shown that orexin deficiency attenuates the ventilator response to hypercapnia and reduces the long-term facilitation of ventilation after exposure to intermitted hypoxia $(21,22)$. In addition, experimental models have shown that orexin $\mathrm{A}$ and $\mathrm{B}$ activate the hypoglossal motor activity and enhance the activity of the largest upper airway dilator muscle: the genioglossus muscle, which is important for maintaining pharyngeal patency $(23,24)$. Eckert et al. reported that a reduction in the activity of such upper airway dilator muscle may be an important contributor mediating REM-related
OSA (25). With respect to previous research, it can be assumed that narcolepsy, especially in NA-CA patients with low or non-detectable levels of orexin, cannot maintain pharyngeal patency, which may lead to an increase occurrence of REM-related OSA. This hypothesis was supported by our results, as the percentage of REM-related OSA in NA-CA with OSA was higher than that in NA w/o CA with OSA (Table 2).

Fig. 2 shows the prevalence of REM-related OSA in NACA and NA w/o CA patients with OSA and of the Japanese OSA sample collected by Sakao et al. (26). The prevalence of REM-related OSA for each definition in NA-CA and NA w/o CA with OSA was higher than that of the Japanese OSA sample. These results might also reflect the pathophysiological characteristics of narcolepsy patients.

REM sleep without atonia has been reported in the patients with narcolepsy, suggesting nocturnal REM sleep motor disturbance in these patients (27). However, our result did not show a significant difference in AHI during REM and NREM sleep between NA-CA and NA w/o CA (Table 3). These results indicated that the pathophysiological mechanism contributing REM-related OSA in the patients with narcolepsy is not fully supported by nocturnal REM sleep motor disturbance. Further studies are needed to con- 


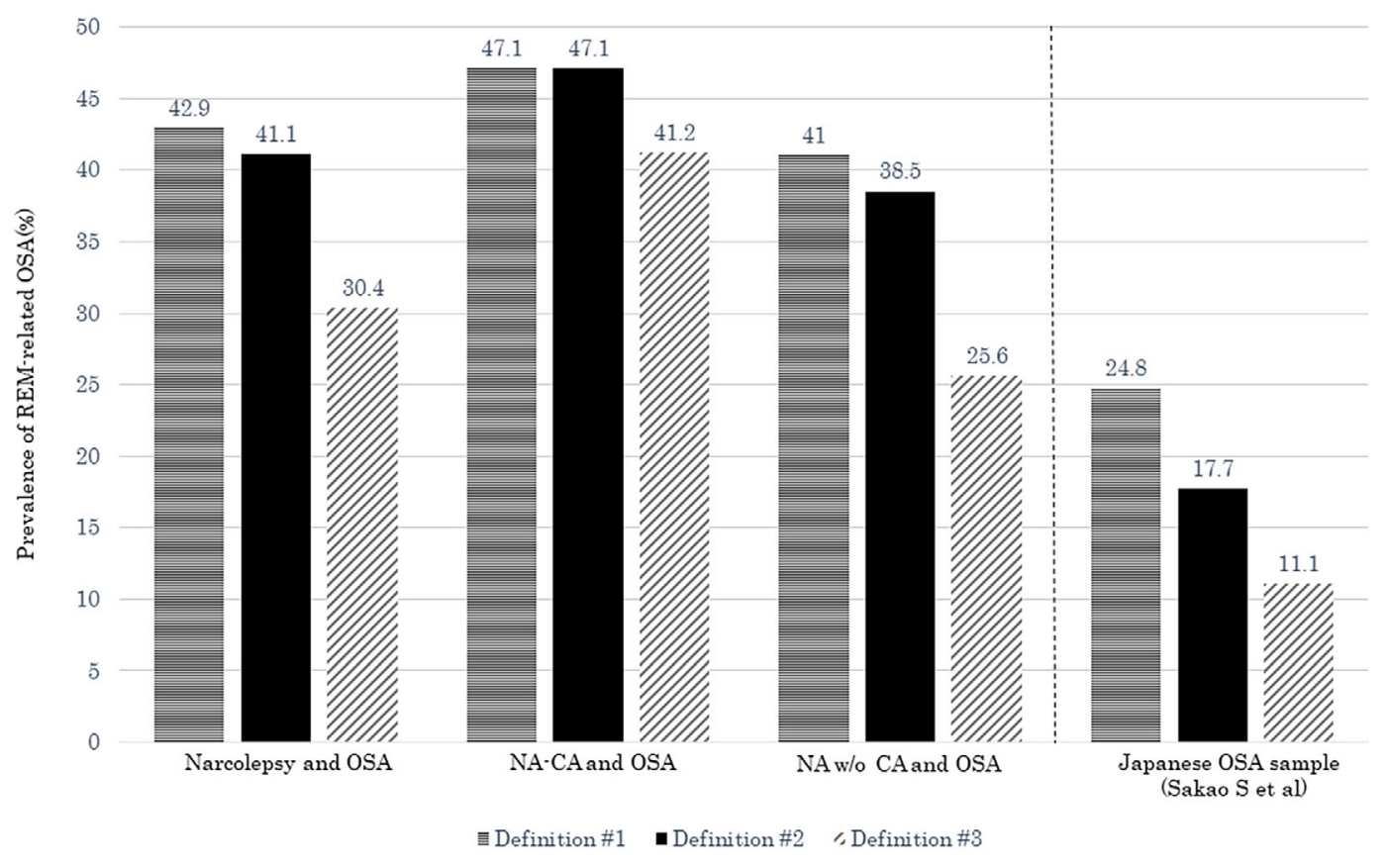

Figure 2. The prevalence of REM-related OSA for each definition in our narcolepsy patients with OSA and Japanese OSA patients reported by Sakao et al. (26). NA-CA: narcolepsy with cataplexy

Table 3. Prevalence of REM-related OSA for Each Definition in Different Studies.

\begin{tabular}{|c|c|c|c|c|c|}
\hline $\begin{array}{c}\text { Study } \\
\text { (Reference) }\end{array}$ & $\begin{array}{l}\text { REM-related } \\
\text { OSA definition }\end{array}$ & Hypopnea definiton & $\begin{array}{l}\text { Defiition of } \\
\text { OSA }\end{array}$ & $\begin{array}{c}\text { OSA } \\
\text { population }\end{array}$ & $\begin{array}{c}\text { Percentage of } \\
\text { REM-related } \\
\text { OSA patients }(\%)\end{array}$ \\
\hline$[18]$ & $\# 1$ & \multirow{2}{*}{ Chicago criteria } & \multirow{11}{*}{$\mathrm{AHI} \geq 5$} & 415 & 36.4 \\
\hline$[28]$ & $\# 2$ & & & 2,486 & 27.5 \\
\hline [29] & $\# 1$ & \multirow{9}{*}{ AASM2007 } & & 100 & 45 \\
\hline [31] & $\# 3$ & & & 931 & 16.8 \\
\hline \multirow{3}{*}{ [19] } & $\# 1$ & & & \multirow{3}{*}{931} & 36.7 \\
\hline & $\# 2$ & & & & 24.4 \\
\hline & $\# 3$ & & & & 13.5 \\
\hline \multirow{3}{*}{ [26] } & $\# 1$ & & & \multirow{3}{*}{468} & 24.8 \\
\hline & $\# 2$ & & & & 17.7 \\
\hline & $\# 3$ & & & & 11.1 \\
\hline \multirow[t]{2}{*}{ [30] } & $\# 2$ & & & 231 & 18.0 \\
\hline & $\# 1$ & & & & 42.9 \\
\hline \multirow[t]{2}{*}{ This study } & $\# 2$ & AASM2007 ver 2.1 & & 56 & 41.1 \\
\hline & $\# 3$ & & & & 30.4 \\
\hline
\end{tabular}

REM: rapid eye movement, OSA: obstructive sleep apnea

firm the external validity of our study results.

Table 3 shows the wide-ranging prevalence of REMrelated OSA in different studies, ranging from $11 \%$ to $45 \%$ of OSA populations $(18,19,26,28-31)$. Such variability in prevalence is due to the usage of different definitions for REM-related OSA. Conwell et al. reported that Definition \# 3 led to a $12.3 \%$ decrease in prevalence when compared to Definition \#2 and a $23.2 \%$ decrease in prevalence when compared to Definition \#1 (19). However, our results did not show a significant decrease in the prevalence when com- paring the relatively strict Definition \#3 with Definitions \#1 and \#2, especially in patients with NA-CA with OSA. REM-related OSA patients in this study satisfied Definition \#3, the most stringent definition, with a higher frequency than in previous reports.

We noted significant differences in the antihypertensive medication use between narcolepsy patients with and without OSA (Table 1). This result might reflect the importance of treating OSA in narcolepsy patients. CPAP treatment is a symptomatic rather than radical treatment; therefore, main- 
taining adherence is crucial to achieve a beneficial effect. However, Sansa et al. reported that $45.4 \%$ of narcolepsy patients who started CPAP treatment could not maintain this treatment (5). Previous studies have reported that CPAP adherence is lower among patients with REM-related OSA than among those with non-stage-specific OSA $(6,9)$. No single factor has been consistently identified as a predictor of CPAP adherence; however, the high prevalence of REMrelated OSA among narcolepsy patients with OSA might affect the results reported by Sansa et al.

Several limitations associated with the present study warrant mention. First, although measuring the cerebrospinal fluid orexin A level is the most important diagnostic criterium for affirming narcolepsy, none of our patients underwent this test, as it is not covered by the Japanese health insurance system (32). This may have led to selection bias. Second, selection bias may have been present, as this study was conducted at a single facility. Finally, the effects of unknown confounding factors could not be excluded because this study was retrospective.

\section{Conclusion}

A high prevalence of REM-related OSA was confirmed in adult narcolepsy patients with OSA. Compared to previous reports, we noted a high frequency of REM-related OSA strictly satisfying Definition \#3. These results might reflect the pathophysiological characteristics of narcolepsy. Further studies are needed to confirm the external validity of our study results.

The authors state that they have no Conflict of Interest (COI).

\section{Acknowledgement}

The authors wish to thank the narcolepsy patients who participated in this study and the specialized clinical engineers who manually scored the PSG and MSLT data, including Youko Murakami, Jyunko Hiraki, Takehiro Yamaguchi, Kaori Kitamura, and Aki Arita.

\section{References}

1. Guilleminault C, Dement WC, Passouant P. Narcolepsy. Spectrum Publications, New York, 1976: 43-56.

2. Roth B. Narcolepsy and hypersomnia: review and classification of 62 personally observed cases. Schweiz Arch Neurol Neurochir Psychiatr 119: 31-41, 1976.

3. Schuld A, Hebebrand J, Geller F, Pollmächer T. Increased body mass index in patients with narcolepsy. Lancet 355: 1274-1275, 2000.

4. Schwartz AR, Patil SP, Laffan AM, Polotsky V, Schneider H, Smith PL. Obesity and obstructive sleep apnea. Proc Am Thorac Soc 15: 185-192, 2008.

5. Sansa G, Iranzo A, Santamaria J. Obstructive sleep apnea in narcolepsy. Sleep Med 11: 93-95, 2010.

6. Almeneessier AS, Almousa Y, Hammad O, et al. Long-term adherence to continuous positive airway pressure in patients with rapid eye movement-only obstructive sleep apnea: a prospective cohort study. J Thorac Dis 9: 3755-3765, 2017.
7. Alzoubaidi M, Mokhlesi B. Obstructive sleep apnea during REM sleep: clinical relevance and therapeutic implications. Curr Opin Pulm Med 22: 545-554, 2016.

8. Grimaldi D, Beccuti G, Touma C, Van Cauter E, Mokhlesi B. Association of obstructive sleep apnea in rapid eye movement sleep with reduced glycemic control in type 2 diabetes: therapeutic implication. Diabetes Care 37: 355-363, 2014.

9. Hoshino T, Sasanabe R, Tanigawa T, et al. Effect of rapid eye movement-related obstructive sleep apnea on adherence to continuous positive airway pressure. J Int Med Res 46: 2238-2248, 2018.

10. Harsh J, Peszka J, Hartwig G, Mitler M. Night-time sleep and daytime sleepiness in narcolepsy. J Sleep Res 9: 309-316, 2000.

11. Kales A, Cadieux RJ, Soldatos CR, et al. Narcolepsy-cataplexy. I. Clinical and electrophysiologic characteristics. Arch Neurol 39: 164-168, 1982.

12. Chokroverty S. Sleep apnea in narcolepsy. Sleep 9: 250-253, 1986.

13. Pataka AD, Frangulyan RR, Mackay TW, Douglas NJ, Rihaet RL. Narcolepsy and sleep-disordered breathing. Eur J Neurol 19: 696702, 2012.

14. Plazzi F, Tartarotti S, Poryazova R, Baumann CR, Bassetti CL. Sleep-disordered breathing and periodic limb movement in narcolepsy with cataplexy: a systematic analysis of 35 consecutive patients. Eur Neurol 70: 22-26, 2013.

15. American Academy of Sleep Medicine. In: International Classification of Sleep Disorders, 2nd ed.: diagnostic and coding manual. American Academy of Sleep Medicine, Westchester, IL, 2005: 7994.

16. Littner MR, Kushida C, Wise M, et al. Practice parameters for clinical use of the multiple sleep latency test and the maintenance of wakefulness test. Sleep 28: 113-121, 2005.

17. Berry RB, Brooks R, Gamaldo CE, et al. The AASM Manual for the Scoring of Sleep and Associated Events: Rules, Terminology and Technical Specifications. Version 2.1, 2014: 16-49.

18. Haba-Rubio J, Janssens JP, Rochat T, Sforza E. Rapid eye movement-related disordered breathing: clinical and polysomnographic features. Chest 128: 3350-3357, 2005.

19. Conwell W, Patel B, Doeing D, et al. Prevalence, clinical features, and CPAP adherence in REM-related sleep-disordered breathing: a cross-sectional analysis of a large clinical population. Sleep Breath 16: 519-526, 2012.

20. Carskadon MA. Guideline for the multiple sleep latency test (MSLT): a standard measure of sleepiness. Sleep 9: 519-524, 1986.

21. Nakamura A, Zhang W, Yanagisawa M, Fukuda Y, Kuwaki T. Vigilance state-independent attenuation of hypercapnic chemoreflex and exaggerated sleep apnea in orexin knockout mice. J Appl Physiol 102: 241-248, 2007.

22. Terada J, Nakamura A, Zhang W, et al. Ventilatory long-term facilitation in mice can be observed during both sleep and wake periods and depends on orexin. J Appl Physiol 104: 499-507, 2008.

23. Zhang GH, Liu ZL, Zhang BJ, et al. Orexin A activates hypoglossal motoneurons and enhances genioglossus muscle activity in rats. Br J Pharmacol 171: 4233-4246, 2014.

24. Dutschmann M, Kron M, Mörschel M, Gestreau C. Activation of Orexin B receptors in the pontine Kölliker-Fuse nucleus modulates pre-inspiratory hypoglossal motor activity in rat. Respir Physiol Neurobiol 159: 232-235, 2007.

25. Eckert DJ, Malhotra A, Lo YL, White DP, Jordan AS. The influence of obstructive sleep apnea and gender on genioglossus activity during rapid eye movement sleep. Chest 135: 957-964, 2009.

26. Sakao S, Sakurai T, Yahaba M, et al. Features of REM-related sleep disordered breathing in the japanese population. Intern Med 54: 1481-1487, 2015.

27. Buskova J, Nevsimalova S, Kemlink D, Sonka K. REM sleep without atonia in narcolepsy. Neuro Endocrinol Lett 30: 757-760, 
2009.

28. Koo BB, Patel SR, Strohl K, Hoffstein V. Rapid eye movementrelated sleep-disordered breathing: influence of age and gender. Chest 134: 1156-1161, 2008.

29. Oksenberg A, Arons E, Nasser K, Vander T, Radwan H. REMrelated obstructive sleep apnea: the effect of body position. J Clin Sleep Med 6: 343-348, 2010.

30. Lee SA, Paek JH, Han SH. REM-related sleep-disordered breathing is associated with depressive symptoms in men but not in women. Sleep Breath 20: 995-1002, 2016.

31. Pamidi S, Knutson KL, Farbod G, Mokhlesi B. Depressive symptoms and obesity as predictors of sleepiness and quality of life in patients with REM-related obstructive sleep apnea: cross-sectional analysis of a large clinical population. Sleep Med 129: 827-831, 2011.

32. Nishino S, Ripley B, Overeem S, Lammers JG, Mignot E. Hypocretin (Orexin) deficiency in human narcolepsy. Lancet 355: 3940, 2000.

The Internal Medicine is an Open Access journal distributed under the Creative Commons Attribution-NonCommercial-NoDerivatives 4.0 International License. To view the details of this license, please visit (https://creativecommons.org/licenses/ by-nc-nd/4.0/).

(C) 2019 The Japanese Society of Internal Medicine Intern Med 58: 2151-2157, 2019 